Article

\title{
How Do Subway Signs Affect Pedestrians' Wayfinding Behavior through Visual Short-Term Memory?
}

\author{
Haoru Li ${ }^{(D)}$, Jinliang $X u^{*}$, Xiaodong Zhang (D) and Fangchen Ma \\ School of Highway, Chang'an University, Xi'an 710064, China; lihaoru93@outlook.com (H.L.); \\ 2017021039@chd.edu.cn (X.Z.); fangchen_ma@outlook.com (F.M.) \\ * Correspondence: xujinliang@chd.edu.cn; Tel.: +86-1370-9208-917
}

check for

updates

Citation: Li, H.; Xu, J.; Zhang, X.; Ma,

F. How Do Subway Signs Affect Pedestrians' Wayfinding Behavior through Visual Short-Term Memory? Sustainability 2021, 13, 6866. https:// doi.org/10.3390/su13126866

Academic Editor: Juan de Oña

Received: 26 March 2021

Accepted: 16 June 2021

Published: 17 June 2021

Publisher's Note: MDPI stays neutral with regard to jurisdictional claims in published maps and institutional affiliations.

Copyright: (c) 2021 by the authors. Licensee MDPI, Basel, Switzerland. This article is an open access article distributed under the terms and conditions of the Creative Commons Attribution (CC BY) license (https:// creativecommons.org/licenses/by/ $4.0 /)$.
Abstract: Recently, subways have become an important part of public transportation and have developed rapidly in China. In the subway station setting, pedestrians mainly rely on visual shortterm memory to obtain information on how to travel. This research aimed to explore the short-term memory capacities and the difference in short-term memory for different information for Chinese passengers regarding subway signs. Previous research has shown that people's general short-term memory capacity is approximately four objects and that, the more complex the information, the lower people's memory capacity. However, research on the short-term memory characteristics of pedestrians for subway signs is scarce. Hence, based on the STM theory and using 32 subway signs as stimuli, we recruited 120 subjects to conduct a cognitive test. The results showed that passengers had a different memory accuracy for different types of information in the signs. They were more accurate regarding line number and arrow, followed by location/text information, logos, and orientation. Meanwhile, information type, quantity, and complexity had significant effects on pedestrians' shortterm memory capacity. Finally, according to our results that outline the characteristics of short-term memory for subway signs, we put forward some suggestions for subway signs. The findings will be effective in helping designers and managers improve the quality of subway station services as well as promoting the development of pedestrian traffic in such a setting.

Keywords: subway; guiding sign; STM capacity; pedestrian cognitive; traffic efficiency

\section{Introduction}

The tertiary structure of memory proposed by Arkinson and Shiffrin in 1968 [1] includes sensory memory, short-term memory (STM), and long-term memory (LTM). Sensory memory is the initial stage of the memory system; then, the information that attracts the individual's attention enters STM. The brain needs a series of coding processes to move from STM to LTM. The tertiary structure of memory is shown in Figure 1.

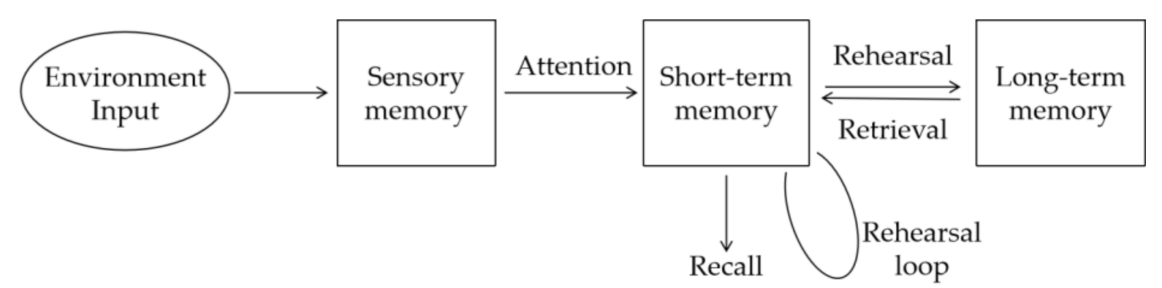

Figure 1. The tertiary structure of memory. (Reprinted from ref. [1]).

Under the rubric of cognitive psychology, STM is an important part of the tertiary structure of memory and is extremely important for performing many everyday tasks [2] In particular, visual short-term memory (VSTM), the holding in mind of visual information for use in current tasks [3], has been a widely researched area in recent years [4]. Many researchers have discussed the storage mechanism of STM. Two general camps have 
emerged: those who believe that the prefrontal cortex mediates both control and storage, and those who believe that the prefrontal cortex largely regulates executive function and that content-specific information is stored primarily in highly selective regions of the early visual cortex [5-8]. Here, we will not review the STM information storage mechanisms. Instead, we will focus on the temporal and capacity properties of STM.

Generally speaking, STM has two commonly recognized features: its short duration and capacity limitations [9]. It has been widely accepted in psychological research that in the case there is no retelling (i.e., the act of repeating the information from previous memory), one's STM usually lasts 5-20 s and no more than 1 min [10,11]. Regarding its capacity limitation, based on the cited studies [12-18], there is still no concrete conclusion. Early estimates of STM capacity by Miller suggested $7 \pm 2$ information objects can be maintained in human STM, and the author proposed that the concept of chunking (the process of combining a number of individual stimuli into meaningful, larger units of information; that is, the organization and recoding of stimuli.) should be used to refer to the basic unit that describes human STM capacity [12]. Cowan et al. re-examined the storage capacity of STM and showed that it revolves around three to five independent information "chunks" or information units $[13,14]$, which is similar to the research results from other researchers. Kaplan found that humans can only remember five major information units, or "chunks" [15]; Luck and Vogel indicated that VSTM capacity was limited to four objects. In their study, participants performed well on a change detection task involving one to three objects, but performance declined rapidly when $4-12$ items were presented [16]. More recent research quantifies iconic memory capacity for complex stimuli as 6.1 items [17], which is significantly higher than traditional previous research [18].

Moreover, researchers have examined whether information type would affect people's STM capacity. Alvarez and Cavanagh found that the capacity varied substantially across the five stimuli classes they examined, ranging from 1.6 for shaded cubes to 4.4 for colors (estimated using a change detection task) [19]. Simon demonstrated that people's STM capacity to memorize phrases and sentences (which were considered as chunks in the study) was slightly smaller than that for words [20]. Yu et al. studied people's STM capacity to memorize Chinese words, and their results were, generally speaking, consistent with the chunking hypothesis of STM; in addition, their tests comprised materials with four different properties (i.e., Chinese characters, graphics, numbers, and positions), and they showed that people's STM capacity was the largest for numbers, followed by graphics, Chinese characters, and positions [21]. A great number of studies on short-term memory have indeed confirmed that one's STM capacity differs according to the properties of the material that needs to be memorized [22,23].

Recently, urban rail transit has been booming in China; hence, people have been gradually learning that the guiding sign systems that are commonly present throughout subway stations play an important role in pedestrians' quick and convenient transit. In 1960, the architect Kevin was already concerned about the importance of guiding signs to provide information for pedestrians in complex environments [24]. Some scholars have put forward that the key factor in designing an effective guidance system for pedestrians is to study their wayfinding behavior. Arthur and Passini linked architecture, signs, and people together and explored the information exchange between space, architecture, and people through a wayfinding test; such procedures allowed them to design the pedestrian guidance system [25]. Nassar considered pedestrians' spatial perception and used simulations to explore what could be the optimal location for such signs [26].

Vilar proposed that pedestrians' wayfinding behavior in daily situations is more rational, so guiding signs are more efficient at guiding pedestrian directions in daily than in emergency situations. This author highlighted the great importance of sign design research in the development of signs for use in daily situations [27]. Moreover, Wang et al. believed that sign visibility was related to attractiveness, the distance between pedestrians and signs, and the occupancy of the signs in pedestrians' field of vision [28]. By using the wayfinding behavior of pedestrians as a theoretical framework, Beck Robert analyzed subway rides 
and studied the signs in this setting from the perspectives of sign location, information content, and redundancy [29].

As can be seen by our descriptions in this section, many researchers have analyzed pedestrians' behavior characteristics related to signs, and some scholars have also examined the influence of specific factors of signs on pedestrian behavior. Nonetheless, these studies have failed to discuss the effectiveness of signage from the perspective of pedestrians' cognitive psychology. When walking in the subway station, pedestrians usually want to achieve their goals as soon as possible, whether they are transferring or going out of the station, especially during the morning or evening rush hours. In the process of wayfinding, in the memory tertiary structure, pedestrians' recognition and memory of signs depends mainly on STM. The information mainly comes in the form of chunks (e.g., line numbers, locations, directions, etc.). We believe that the theory of STM should be combined with the subway signs to comprehensively and extensively analyze the cognitive process inherent in pedestrians in this context. This knowledge may be the key to producing highly effective signs, which can greatly improve pedestrian travel efficiency.

Hence, this study aimed to examine whether information complexity affects pedestrians' STM capacity in a subway station setting and to clarify what types of information chunks are more conducive to pedestrians' effective memorization of information. In particular, we adopted the concepts of information chunks and of visual information to describe which visual features of the signs could be stored in pedestrians' STM. Nonetheless, we did not mean to refer to information in its mathematical sense, which is the classical form utilized in information-theory-based studies. Alvarez et al. found that information search rates affected STM, suggesting that the more information the sign contained, the fewer items people would remember [19]. Therefore, from another perspective, we also examined whether people's memory capacity related to the total number of information chunks contained in the sign; to do so, we observed pedestrians' STM ability for memorizing signs that contained different numbers of information chunks at the same time interval. We hypothesized that people tend to remember simpler information; namely, with the increase in the number of information chunks in a sign, pedestrians' memory may become disturbed, resulting in the reduction of STM capacity.

Based on the above concepts and assumptions, this paper conducted a cognitive memory test on 120 subjects by selecting real pictures of subway station signs in China. The test results showed that passengers had a different memory accuracy for different types of information in the signs. Meanwhile, information type, quantity, and complexity had significant and differential effects on pedestrians' STM capacity. In this study, the information chunking theory in cognitive psychology was applied to the study of pedestrian cognition and memory of subway signs. Through detailed analysis and classification of the entrance and exit sign information, the STM characteristics of pedestrians using subway signs are discussed, and the STM capacity is explored. The findings will be effective in helping designers and managers to improve the quality of the subway station services as well as promoting pedestrian traffic and sustainable subway development. As "Caring for the earth: A Strategy for Sustainable Living" points out, "the ultimate foothold of sustainable development is human society, namely to improve the quality of human life and create a better living environment".

The paper is organized as follows. The test method, the selection of stimuli, and the data pre-processing method are presented in Section 2. Based on the characteristics of the entrance and exit sign information, Section 3 describes the pedestrians' STM characteristics in response to these signs. The research is discussed in Section 4 and summarized in Section 5 .

\section{Materials and Methods}

\subsection{Study Subjects}

People vary greatly in their individual cognitive and memory characteristics. In this paper, all potential subway passengers were considered as research objects. In order to 
ensure the scientific validity and comprehensiveness of the cognitive memory test, the selection of test samples should conform to the principle of statistical sample selection, and a certain sample size must be guaranteed. Hence, we utilized a sampling survey method when selecting subjects for our test, and the sample size extracted by this method can be determined according to the results of the following equation [30].

$$
N \geq\left(\frac{S K}{E}\right)^{2}
$$

where $N$ is the sample size; $S$ is the sample standard deviation, $S=0.5 ; K$ is a constantwhen the confidence is equal to $95 \%, K=1.96$; and $E$ is the subject personnel memory capacity allowable error, $E=0.1$.

According to the above equation, the sample size for our study needed to be greater than 96. Considering that there are many types of subway passengers (gender composition, age composition, etc.), we expanded the required sample size to 120 . The only exclusion criteria for this study was being under the age of 12; this was because young children are often accompanied by adults when traveling, so they lack the ability to recognize signs independently.

For the subject selection, we adopted the method of social wide recruitment. In and out of the subway station, we asked the pedestrians if they would like to cooperate with us in the paid test and told them the test lasted about $20 \mathrm{~min}$ and each person would be paid 100 RMB for their time. After the subject agreed, we would confirm with him/her when and where the test would be conducted. Before the test, the subjects were told that the test was anonymous and the results were only for academic research. All subjects gave their informed consent for inclusion before they participated in the study.

Finally, the age distribution of the subjects we collected ranged from 15 to 76 years (mean age 34.9 years), with the sex ratio of the subjects close to the sex ratio of China's population. The subjects selected in the paper cover various types of pedestrians, including those who often take subway, occasionally take subway, and those who have never taken subway.

\subsection{Stimuli}

To make the signs used in the test consistent with the signs used in real subway stations-i.e., make the test reflect, as much as possible, the real scenario pedestrians would see in a subway station-we investigated all the subway lines in Beijing, Shanghai, Guangzhou, and Xi'an that were older and complete in their development. Through this procedure, we collected a large number of photographs of subway signs, and the photos were taken from a pedestrian vision perspective. These photos were selected and used in the test of this study through projection. To ensure whether the size of information affects people's memory, we referred to the literature on cognitive psychology. Scholars pointed out that the size of information has little effect on people's memory, under the condition that the size difference is small and people can clearly identify the information [31]. We found that subway signs can be of a hanging type, ground type, attached type, and standing type. The information types in the signs were different based on their roles (entrance, exit, or transfer). Sample subway sign types are shown in Figure 2. The hanging signs were the most common, and the entrance sign and exit sign were the necessary signs in every subway station, which were related to whether pedestrians can enter the subway quickly and accurately or find the correct exit to leave. Therefore, we utilized pictures of entrance and exit hanging signs as the study stimuli.

Our test used the concept of information chunk to partition the information contained in the signs. Take Figure 3 as an example, which serves to show the chunk implementation method we utilized for the subway hanging signs. 


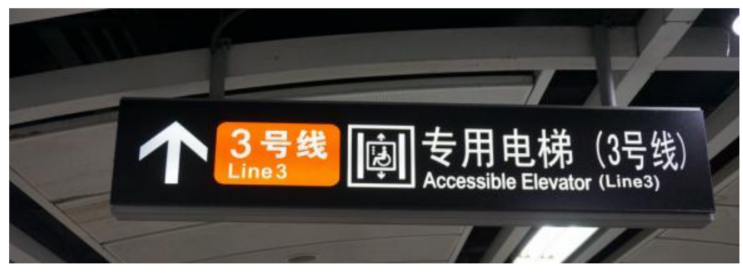

(a) Hanging type (transfer)

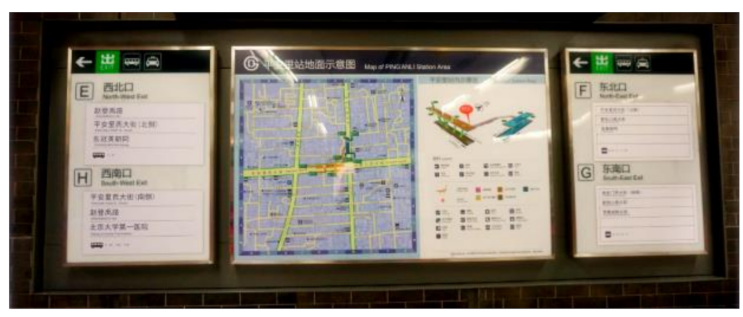

(c) Attached type (exit)

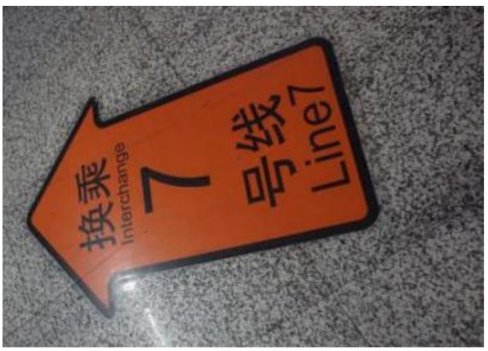

(b) Ground type (transfer)

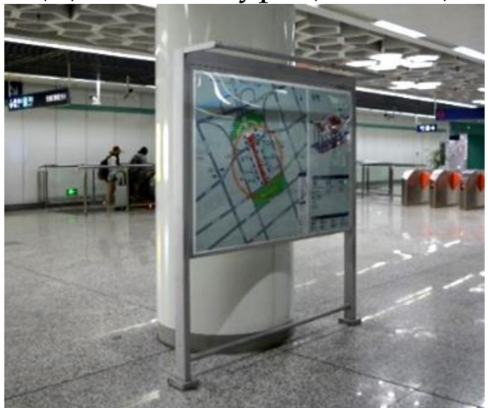

(d) Standing type (exit)

Figure 2. Subway sign types.

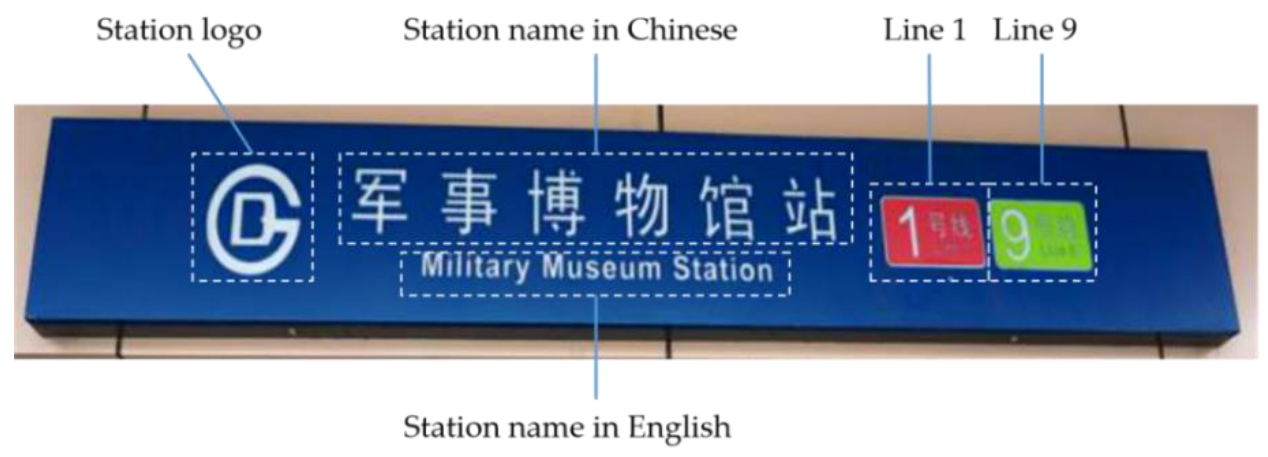

(a) Five information chunks - hanging sign (entrance)

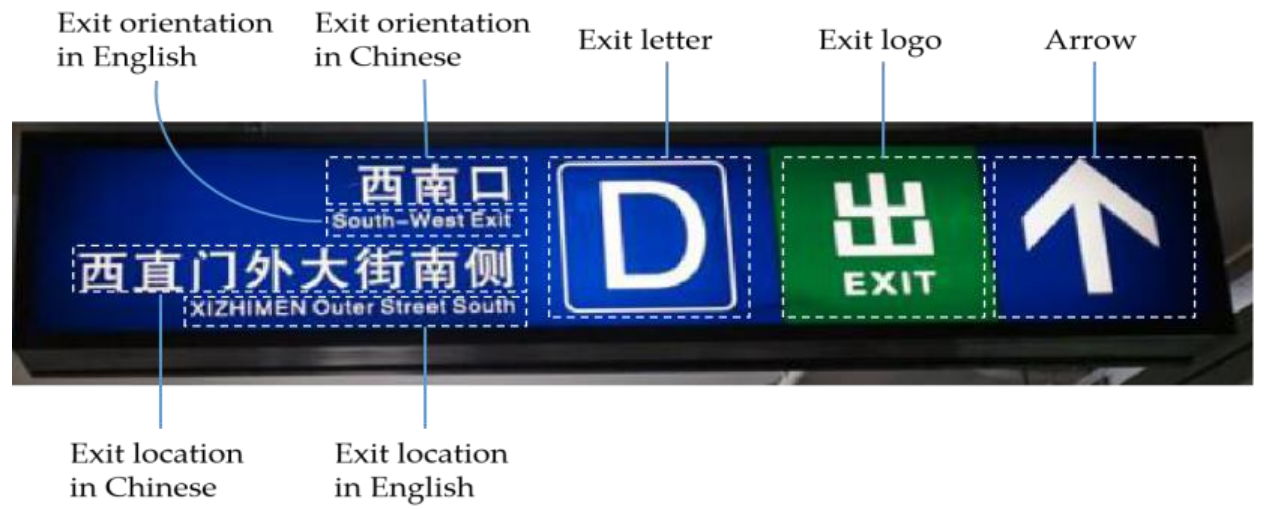

(b) Seven information chunks - hanging sign (exit)

Figure 3. Subway sign types.

The sign shown in Figure 3a is located in the entrance of the subway station and contains 5 information chunks: the logo of the Beijing subway station, the phrase "military museum station" in Chinese, the phrase "military museum station" in English, information 
on line 1, and information on line 9. In Figure 3b, the sign is located in the exit of the subway station and contains 7 information chunks: the phrase "South-West Exit" in both Chinese and English, the phrase "XIZHIMEN Outer Street South" in both Chinese and English, the " $\mathrm{D}$ " marking the exit letter of the Beijing subway, an exit logo and wording that is standardized in China for all subway stations, and an arrow indicating a direction.

The hanging signs collected were divided into two categories: the entrance signs that guide pedestrians into the station and the exit signs that guide pedestrians out of the station. The signs were then subdivided into four groups according to the number of chunks they contained: 4-chunk, 5-chunk, 6-chunk, and 7-chunk. Each group contained four signs corresponding to the four memory time intervals ( $5 \mathrm{~s}, 10 \mathrm{~s}, 20 \mathrm{~s}, 60 \mathrm{~s})$ mentioned in Section 2.4. Hence, 16 entrance signs and 16 exit signs were selected. In this test, a total of 32 subway suspension signs were studied.

According to the statistical classification of the collected sign information, the entrance sign generally contains the following information: (1) exit number, (2) line number, (3) subway station logo, (4) station name in Chinese, and (5) station name in English. The export sign generally contains the following information: (1) exit letters, (2) exit in Arabic numerals, (3) exit orientation, (4) exit in Chinese, (5) national standardized exit logo, (6) direction arrow, (7) exit location information in Chinese, and (8) location information in English. The analysis of pedestrian's memory characteristics for the information contained in signs is based on the above information classification.

\subsection{Preliminary Test}

A preliminary test was put forward to check whether the chunk method used in the test is feasible and accurate. The pre-test was very simple, requiring only one test operator. A total of 20 students were recruited on campus and taken to the test office one by one (the rest waited outside the lab). The test material was one randomly selected sign from among the 32 signs mentioned in Section 2.2. The subject was shown the subway sign using a projector and asked, "describe all the information you saw". Then the test operator recorded the response in the preliminary test record sheet. The sheets corresponding to each sign were different. Table 1 shows the test record sheet corresponding to Figure 2a.

Table 1. Preliminary test record sheet.

\begin{tabular}{cc}
\hline Information Chunks & Obtained Information \\
\hline Subway sign & \\
Station name in Chinese & \\
Station name in English & \\
Line 1 \\
Line 9
\end{tabular}

The results of the preliminary test were basically in line with expectations, and the way the subjects described the information was consistent with the information chunk method in this paper. However, the English information was excluded: 16 of the 20 subjects completely ignored the English information, 2 subjects asked whether they needed to describe the English information after describing all the information, and only 2 subjects accurately described the English information.

According to the results of the preliminary test, the subjects tended to ignore the repetitive information in the non-native language. Hence, we adjusted the counting form of sign information chunks and ignored the English information. For the 32 flags we picked earlier, we recalculated the number of information chunks they contained. The number of information chunks in the four test groups was adjusted from 4-chunk, 5-chunk, 6-chunk, and 7-chunk to 3-chunk, 4-chunk, 5-chunk, and 6-chunk. The number of signs in each test group were regulated to 4 by deleting the redundant signs or adding additions signs. Based on this principle, 32 signs comprised the final set of the formal test stimuli. 


\subsection{Formal Test}

The formal test was conducted in the same office as the test site for the preliminary test. Based on our aims, the dependent variable in this test was time interval, and the independent variable was the number and type of information chunks that were accurately obtained by different subjects at a certain time interval without repeating the display of the signs. Considering the time characteristics of STM shown by previous research, the retention time revolves around 5-20 s without retelling/repeating the presentation, but it does not extend for more than $1 \mathrm{~min}$. Hence, in our test, we set the time points at $5 \mathrm{~s}, 10 \mathrm{~s}$, $20 \mathrm{~s}$, and $60 \mathrm{~s}$.

Additionally, to ensure that all procedures related to the STM test were carried out without repetition, we referenced the Brown-Peterson method [32]. We included interference in between stimuli presentations. Subjects were asked to perform quick (the quickness is important to prevent people from revisiting their memories) two-digit unordered calculations involving addition and subtraction.

To ensure that the sign was the only variable, all the subjects watched the stimuli in exactly the same order, gradually increasing from three to six chunks, and from $5 \mathrm{~s}$ to $60 \mathrm{~s}$ in each chunk group.

To test the large number of subjects, we took 6 days to do the test. Each day, 20 subjects were tested in the morning and afternoon. Before the test, all the subjects were informed they could not communicate about the test. In particular, the following procedures were undertaken during the test, controlled by 2 operators:

1. One subject was asked to enter the room and sit ten meters directly in front of the projector screen, while the rest waited outside. Operator 1 used the projector to display the subway sign photo (e.g., Figure 1).

2. About $10 \mathrm{~s}$ later, the subject was asked by Operator 2 if she/he had obtained all the information contained in the sign and had drawn " $\sqrt{ }$ " in the "obtained information" column. Then, Operator 1 turned off the monitor, Operator 2 calculated the number of information chunks in the sign and filled the number in the table (e.g., the 4 information chunks in Figure 1a).

3. Then, subjects were asked by Operator 1 to perform a two-digit unordered addition and subtraction until the interval corresponding to the sign was over (the time interval corresponding to each sign was marked in the table, e.g., see Table 2).

Table 2. Formal test record sheet.

\begin{tabular}{ccc}
\hline Information Chunks & Obtained Information & Accurate Remember \\
\hline Subway sign & & \\
Station name in Chinese & \\
Station name in English & \\
Line 1 & \\
Line 9 & \\
Total information amount & \\
Short-term memory capacity after $20 \mathrm{~s}$ & \\
\hline
\end{tabular}

4. Then, Operator 2, who was recording the data, asked subjects what information they could recall from the sign. For the information chunks that the subject could clearly recall, the worker drew a " $\sqrt{ }$ " in the "Accurate Remember" column. Based on this process, the operator could calculate the number of chunks that subjects were able to recall with clarity, and the total number was filled in the "Short-term memory capacity" line.

5. Then, steps 1-4 were repeated until all the 32 signs had been tested.

6. The next subject was let into the room and steps $1-5$ were repeated.

7. At the end of the test, the operator asked temporary questions about the test, such as if the subject had always ignored some information types and why. 


\subsection{Data Analysis}

In total, data from 106 subjects were effectively collected; 14 subjects didn't complete all the sign tests, and the collection rate was $88.3 \%$. In total, 51 were men and 55 were women, and the male-female ratio of the subjects was close to the sex ratio of China's population. Subjects' age ranged from 17 to 76 .

In this paper, four information chunks were analyzed for the entrance signs: (1) subway line number (letter or Arabic numeral), (2) subway logo, (3) the station name, and (4) the exit number. There were seven types of information chunks in exit signs: (1) exit letters, (2) exit in Arabic numerals, (3) exit orientation, (4) exit in Chinese, (5) the national standardized logo, (6) the direction arrow, and (7) the exit location information in Chinese.

After the test, we counted the number of people who were able to accurately remember the information chunk contained in the entrance signs and exit signs (hereafter accurate memory population) and calculated the accurate memory population ratio (R) (Ratio of the accurate memory population to the total number of 106 participants, expressed as a percentage) to explore the impact of different information types and information chunk sizes on pedestrians' STM. The calculation formula was as follows:

$$
R=\left(\frac{P_{1}}{P}\right)
$$

where: $P_{1}$ is the accurate memory population and $P$ is the total number of subjects.

For signs that contained more than one chunk of the same type of information, we calculated the mean to analyze the accurate memory population ratio for that type of chunk, and the statistical results are shown in Tables 3 and 4.

Table 3. Different information chunk accurate memory population ratio (entrance sign).

\begin{tabular}{|c|c|c|c|c|c|}
\hline \multirow{2}{*}{$\begin{array}{c}\text { Number of } \\
\text { Information Chunks }\end{array}$} & \multirow{2}{*}{ Information Type } & \multicolumn{4}{|c|}{ Time Interval (s) } \\
\hline & & 5 & 10 & 20 & 60 \\
\hline \multirow{4}{*}{ 3-chunk } & Line No. & $92.45 \%$ & $90.57 \%$ & $91.51 \%$ & $85.85 \%$ \\
\hline & Station name & $91.51 \%$ & $90.57 \%$ & & $76.42 \%$ \\
\hline & Subway logo & $43.40 \%$ & & $43.40 \%$ & $39.62 \%$ \\
\hline & Exit No. & & $80.19 \%$ & $79.25 \%$ & \\
\hline \multirow{4}{*}{ 4-chunk } & Line No. & $81.13 \%$ & $80.19 \%$ & $78.30 \%$ & $76.42 \%$ \\
\hline & Station name & $73.58 \%$ & $73.58 \%$ & $68.87 \%$ & $63.21 \%$ \\
\hline & Subway logo & $37.74 \%$ & $34.91 \%$ & $34.91 \%$ & $30.19 \%$ \\
\hline & Exit No. & $72.64 \%$ & $69.81 \%$ & $67.92 \%$ & $63.21 \%$ \\
\hline \multirow{4}{*}{ 5-chunk } & Line No. & $78.30 \%$ & $75.47 \%$ & $73.58 \%$ & $67.92 \%$ \\
\hline & Station name & $64.15 \%$ & $62.26 \%$ & $61.32 \%$ & $53.77 \%$ \\
\hline & Subway logo & $33.96 \%$ & $31.13 \%$ & $27.36 \%$ & $23.58 \%$ \\
\hline & Exit No. & $65.09 \%$ & $63.21 \%$ & $63.21 \%$ & $51.89 \%$ \\
\hline \multirow{4}{*}{ 6-chunk } & Line No. & $71.70 \%$ & $65.09 \%$ & $56.60 \%$ & $45.28 \%$ \\
\hline & Station name & $62.26 \%$ & $56.60 \%$ & $52.83 \%$ & $46.23 \%$ \\
\hline & Subway logo & $32.08 \%$ & $30.19 \%$ & $27.36 \%$ & $22.64 \%$ \\
\hline & Exit No. & $43.40 \%$ & $40.57 \%$ & $33.02 \%$ & $25.47 \%$ \\
\hline
\end{tabular}

To further analyze pedestrians' memory patterns for different types of information, all information chunks were summarized and categorized into six information types: (1) numerals (e.g., exit number, line number), (2) letters (e.g., exit letter, line letter), (3) orientation in Chinese, (4) location in Chinese, and (5) direction arrow. Then, we calculated the memory population ratio (R) for each information type. Through the analysis of the ratio, we can more intuitively explore the STM changes for different types of information at different time intervals.

Based on the above data analysis method, the characteristics of pedestrians' memory for entrance and exit signs can be further analyzed. 
Table 4. Different information chunk accurate memory population ratio (exit sign).

\begin{tabular}{|c|c|c|c|c|c|}
\hline \multirow{2}{*}{$\begin{array}{c}\text { Number of } \\
\text { Information Chunks }\end{array}$} & \multirow{2}{*}{ Information Type } & \multicolumn{4}{|c|}{ Time Interval (s) } \\
\hline & & 5 & 10 & 20 & 60 \\
\hline \multirow{7}{*}{ 3-chunk } & Exit letter & & & & \\
\hline & Exit No. (numeral) & $89.62 \%$ & & & \\
\hline & Exit orientation & & & & \\
\hline & Exit in Chinese & $94.34 \%$ & $92.45 \%$ & $88.68 \%$ & $85.85 \%$ \\
\hline & Logo & & & $44.34 \%$ & $37.74 \%$ \\
\hline & Arrow & $92.45 \%$ & $91.51 \%$ & $87.74 \%$ & \\
\hline & Place in Chinese & & $87.74 \%$ & & $84.91 \%$ \\
\hline \multirow{6}{*}{ 4-chunk } & Exit letter & & $61.32 \%$ & $59.43 \%$ & \\
\hline & Exit No.(numeral) & $82.08 \%$ & & & $76.42 \%$ \\
\hline & Exit orientation & & & $49.06 \%$ & \\
\hline & $\begin{array}{c}\text { Exit in Chinese } \\
\text { Logo }\end{array}$ & $86.79 \%$ & $82.08 \%$ & & $72.64 \%$ \\
\hline & Arrow & $85.85 \%$ & $83.02 \%$ & $79.25 \%$ & $74.53 \%$ \\
\hline & Place in Chinese & $81.13 \%$ & $73.58 \%$ & $67.92 \%$ & $54.72 \%$ \\
\hline \multirow{7}{*}{ 5-chunk } & Exit letter & & $51.89 \%$ & & $41.51 \%$ \\
\hline & Exit No.(numeral) & $81.13 \%$ & & $73.58 \%$ & \\
\hline & Exit orientation & & $45.28 \%$ & & \\
\hline & Exit in Chinese & $76.42 \%$ & & & $53.77 \%$ \\
\hline & Logo & & $38.68 \%$ & $34.91 \%$ & \\
\hline & Arrow & $84.91 \%$ & $79.25 \%$ & $76.42 \%$ & $70.75 \%$ \\
\hline & Place in Chinese & $77.36 \%$ & $74.53 \%$ & $58.49 \%$ & $38.68 \%$ \\
\hline \multirow{7}{*}{ 6-chunk } & Exit letter & $48.11 \%$ & $40.57 \%$ & $29.25 \%$ & \\
\hline & Exit No.(numeral) & & & & $47.17 \%$ \\
\hline & Exit orientation & $38.68 \%$ & $33.02 \%$ & $23.58 \%$ & \\
\hline & Exit in Chinese & & $60.38 \%$ & $50.94 \%$ & $45.28 \%$ \\
\hline & Logo & & & $26.42 \%$ & $17.92 \%$ \\
\hline & Arrow & $64.15 \%$ & $60.38 \%$ & $57.55 \%$ & $52.83 \%$ \\
\hline & Place in Chinese & $51.89 \%$ & $50.94 \%$ & & $34.91 \%$ \\
\hline
\end{tabular}

We hypothesized that people would have different STM capacities for signs that contained different types of information. Then, based on the data collected in Table 2, the average STM capacity of 106 subjects at each interval for each information chunk amount was calculated. The results are shown in Table 5.

Table 5. Short-term memory capacity.

\begin{tabular}{cccccc}
\hline \multirow{2}{*}{ Sign Type } & \multirow{2}{*}{$\begin{array}{c}\text { Information } \\
\text { Chunks }\end{array}$} & $\mathbf{5}$ & $\mathbf{1 0}$ & $\mathbf{2 0}$ & $\mathbf{6 0}$ \\
\cline { 3 - 5 } & 3-chunk & 2.27 & 2.61 & 2.14 & 2.02 \\
\multirow{3}{*}{ Entrance sign } & 4-chunk & 2.65 & 2.58 & 2.50 & 2.33 \\
& 5-chunk & 3.20 & 3.08 & 2.99 & 2.65 \\
& 6-chunk & 3.53 & 3.23 & 2.83 & 2.44 \\
\hline \multirow{3}{*}{ Exit sign } & 3-chunk & 2.76 & 2.72 & 2.21 & 2.08 \\
& 4-chunk & 3.36 & 3.00 & 2.56 & 2.78 \\
& 5-chunk & 3.20 & 2.90 & 2.43 & 2.05 \\
& 6-chunk & 3.19 & 2.96 & 2.45 & 2.33 \\
\hline
\end{tabular}

\section{Results}

To ensure the universality of the selected samples, we conducted the test on various types of pedestrians. The difference analysis was conducted on the memory of pedestrians with different degrees of familiarity and different age groups. The analysis results showed that there was no significant difference in the ability to remember information, regardless of the situation of taking the subway (Sig. $>0.05$ ). At the same time, except for older subjects 
(over 60 years old), there was no significant difference in the memory of information among age groups.

\subsection{Entrance Hanging Sign Basic Characteristics}

To better reflect pedestrians' STM of the four types of information chunks, we utilized $\mathrm{R}$ as the variable and drew the accurate memory population ratio histogram by time, the four types of signs, and the information chunks (shown in Figure 4).

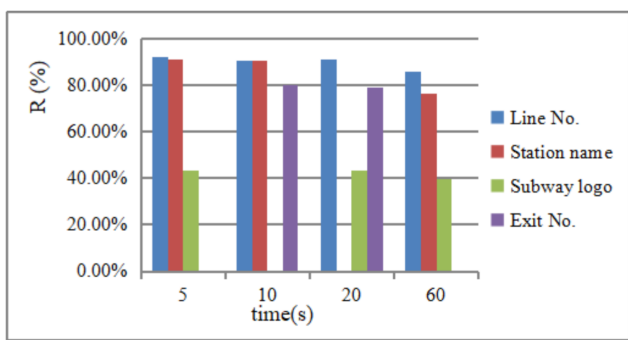

(a) 3-chunk entrance hanging sign

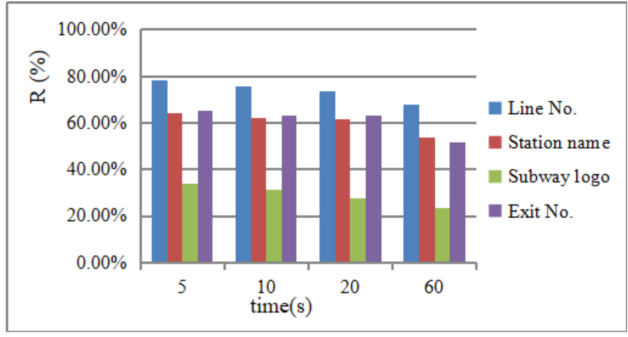

(c) 5-chunk entrance hanging sign

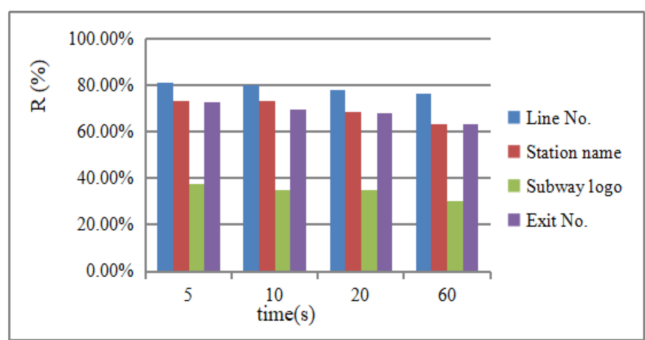

(b) 4-chunk entrance hanging sign

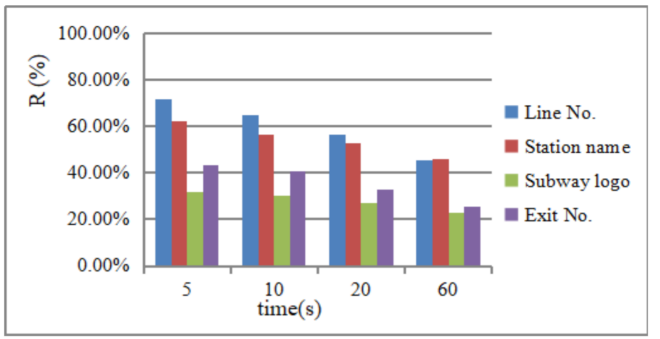

(d) 6-chunk entrance hanging sign

Figure 4. Entrance hanging sign R-time statistical figures.

For the 3-chunk entrance hanging signs, all the signs had exit and line numbers, and some of the signs had the subway station logo, while others had the station name. For the 4-, 5-, and 6-chunk signs, they usually contained all four types of information chunks. Our results showed that $\mathrm{R}$ for the 6-chunk entrance signs had a diminishing curve that was different from that of the other sings: With the increase in time, the accurate memory population ratio showed an obvious "step-by-step" decline. For line No. information in the 6-chunk group, from $5 \mathrm{~s}$ to $60 \mathrm{~s}$, the change of $\mathrm{R}$ was $71.70 \%$ to $65.09 \%$ to $56.60 \%$ to $45.28 \%$, for station name it was $62.26 \%$ to $56.60 \%$ to $52.83 \%$ to $46.23 \%$, for station $\log$ it was $32.08 \%$ to $30.19 \%$ to $27.36 \%$ to $22.64 \%$, and for exit No. it was $43.40 \%$ to $40.57 \%$ to $33.02 \%$ to $25.47 \%$. Nonetheless, for the 3-, 4-, and 5-chunk entrance signs, the R for the four types of information did not significantly change overtime. The biggest decline of $\mathrm{R}$ related to the line No. in the 6-chunk hanging signs, which decreased $26.42 \%$ from $5 \mathrm{~s}$ to $60 \mathrm{~s}$.

Then, Pearson correlation analysis was performed between time interval and $\mathrm{R}$ in each information chunk group using SPSS. It was found that R was significantly negatively correlated with the interval time ( $r>0.900$, Sig. $<0.05)$.

Among the four different types of information chunks (line number, station name, subway logo, and exit number), the four entrance hanging sign groups, and during any interval of time, $\mathrm{R}$ was the largest for the line number, followed by the station name, exit number, and subway logo. After the test, the operator asked subjects why they often ignored the signs, and most pedestrians said that the subway logo was not necessary information, so they often ignored it. Based on our results, the overall rule was as follows: with the increase in the number of information chunks contained in the signs, the $R$ decreased at every time interval.

Then, for different time interval, the number of information chunks contained in the signs and the different types of information chunks were tested for correlation analysis. We found that 10 of the 16 results showed strong negative correlations $(r>0.900$, 
Sig. < 0.05), while the remaining six showed similar strong correlations but low significance $(r>0.900$, Sig. $>0.05)$. According to the analysis of the data, it was found that this was due to the high drop in the pedestrian's memory rate for numbers and logo information in the 5-and 6-chunk groups. In general, the accurate memory population ratio of each type of information in entrance signs decreased significantly with the increase of the number of information chunks.

\subsection{Exit Sign Basic Characteristics}

In order to better reflect pedestrians' STM of the seven types of information chunks present in exit signs, the accurate memory population ratio by time, information chunk, and type of sign is shown in Figure 5.

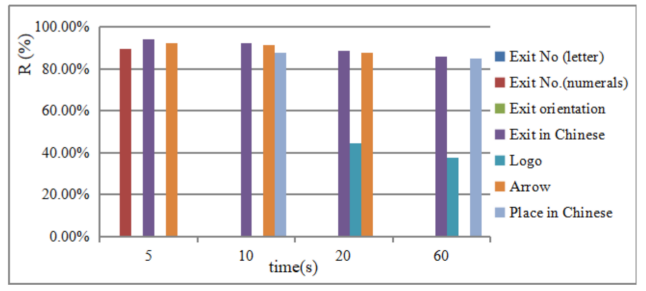

(a) 3-chunk exit hanging sign

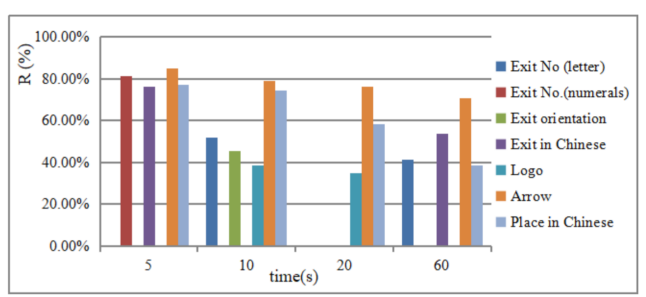

(c) 5-chunk exit hanging sign

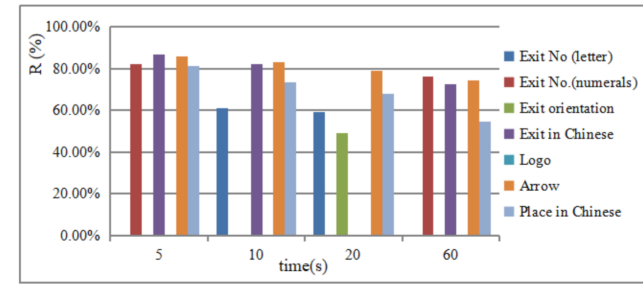

(b) 4-chunk exit hanging sign

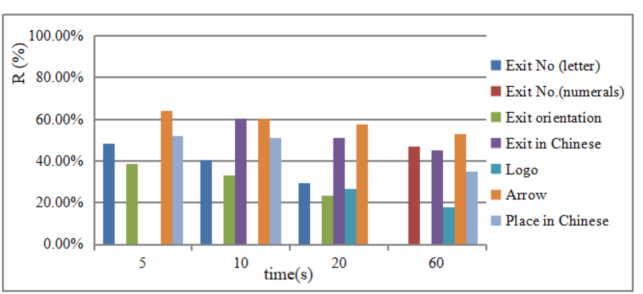

(d) 6-chunk exit hanging sign

Figure 5. Exit hanging sign R-time statistical figure.

Among the seven types of information chunks we analyzed, direction arrows and locations were the most common types of information chunks in the signs. Of the 16 signs tested, 15 contained direction arrows and 13 contained locations. Moreover, and generally speaking, the signs had at least one of the exit in Chinese or the exit logo. Of the 16 signs tested, 12 contained the exit in Chinese and six contained the exit logo. Some subway stations tended to use Arabic numerals to number exits, while others used letters. In a few areas, subway stations showed the exit orientation in the exit signs.

When analyzing short-term memory for exit signs, we found that the results differed from those of the entrance signs. The $R$ decreased with time in the 4-, 5-, and 6-chunk exit hanging signs, showing a step-like decline. The data of each information chunk group were analyzed by SPSS.

It was found that the accurate memory population ratio was significantly correlated with interval time in the 4-, 5-, and 6-chunk exit hanging sign groups ( $r>0.900$, Sig. < 0.05). Nonetheless, in the 3-chunk group, the $\mathrm{R}$ was not significantly correlated with interval time $(r=0.091$, Sig. $>0.05)$. This may be because, generally, the exit signs contained too many types of information chunks, so when the number of information chunks was small, the data for each type of information was small, which led to the inability to reflect better correlation.

Among the different types of information chunks in the exit sign, the $\mathrm{R}$ was the largest for the exit in Arabic numerals, the exit in Chinese, and the direction arrow, followed by the exit location information, exit letters, and the exit orientation and the exit logo. Corroborating what happened with the subway logo in the entrance hanging sign, the stair logo, exit logo, and the other logos present in the exit signs were easily ignored. Once more, 
similar to the results for the entrance sign, $\mathrm{R}$ was significantly correlated with the number of information chunks for each type of information chunk contained in the exit sign at all the time intervals $(5 \mathrm{~s}, 10 \mathrm{~s}, 20 \mathrm{~s}$, and $60 \mathrm{~s})$ (Sig. < 0.05).

\subsection{Pedestrian STM for Subway Signs}

Based on our results, subjects' memories for different types of information differed individually. Subjects' $R$ trend for each type of information chunk by time is shown in Figure 6.

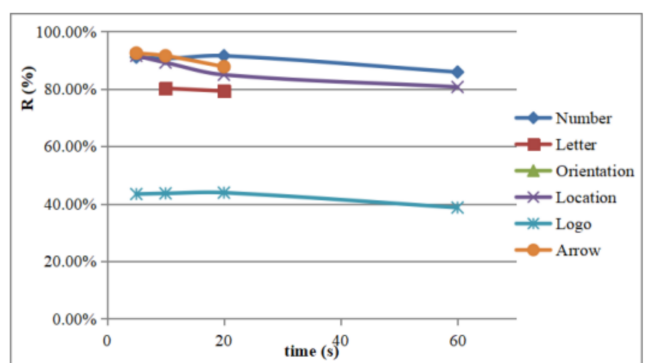

(a) 3-chunk

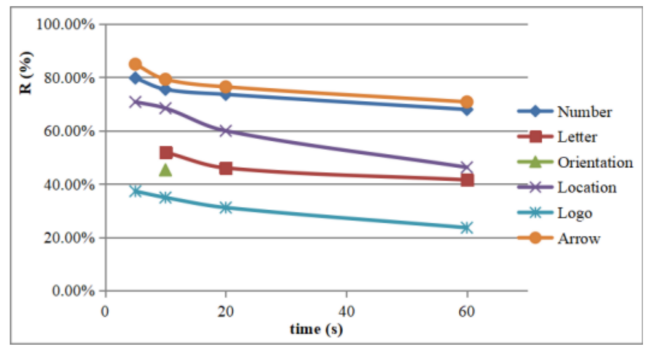

(c) 5-chunk

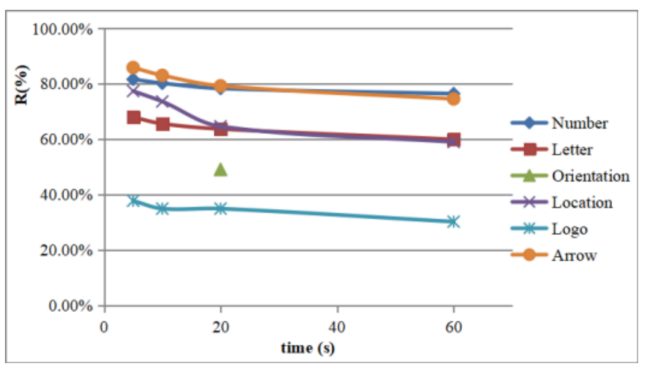

(b) 4-chunk

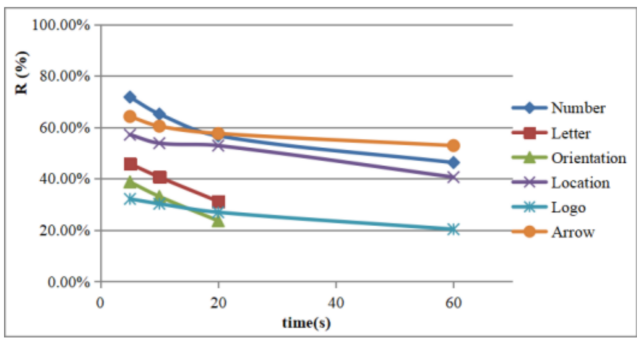

(d) 6-chunk

Figure 6. R of different types of information chunks over time.

Generally speaking, the order, from high to low, of people's ability to accurately remember different types of information chunks was as follows: numerals $\approx$ arrow $>$ location $>$ letter $>\operatorname{logo} \approx$ orientation. Moreover, people were more likely to remember exits that were numbered than exits that used letters. Regarding orientation information, pedestrians were shown to have poor memory and to easily overlook information.

Based on Figure 5, it can be seen that the $\mathrm{R}$ of each information chunk changed differently with time. The $\mathrm{R}$ of number, arrow, and logo information decreased slightly in 20-60 s, while the $\mathrm{R}$ of location information decreased a little faster. The $\mathrm{R}$ for the 4-, 5-, and 6-chunk signs showed the following common characteristics: the slope of 11 of the 13 complete curves, from $5 \mathrm{~s}$ to $60 \mathrm{~s}$, was larger in the range of 5-10 s, indicating that the overall forgetting speed of the information chunk reaches its peak before $10 \mathrm{~s}$. Moreover, there were 14 complete curves in the $10-60 \mathrm{~s}$ period, among which 12 had a higher slope in the 10-20 s period than that in the 20-60 s period; this further indicates that subjects' forgetting rate for various types of information chunks was faster at the beginning of the test and slower at the end.

\subsection{Pedestrians' STM Capacity for Subway Signs}

Based on the results in Table 5 , we show subjects' memory capacity by type of sign (entrance or exit sign), information chunk, and time in Figure 7.

By comparing the number of information chunks contained in the entrance and the exit signs, it can be seen that the information in entrance signs is relatively simple (the entrance sign usually contained four information types, while in the exit sign, we compiled seven common types of information); this is even more truthful when the amount of information chunks increases. 
Subjects' STM capacity did not increase with the increase in the number of chunks of information contained in signs. When the type of information in the sign was relatively simple (entrance sign), subjects' maximum STM capacity at $5 \mathrm{~s}, 10 \mathrm{~s}, 20 \mathrm{~s}$, and $60 \mathrm{~s}$ was 3.53 , $3.23,2.83$, and 2.44, respectively (all four results appeared when analyzing the 5- and 6chunk hanging signs). Therefore, our results showed that, the more information chunks in a sign, the greater the pedestrians' STM capacity. Nonetheless, subjects' memory capacities when analyzing 6-chunk signs at $20 \mathrm{~s}$ and $60 \mathrm{~s}$ were lower than their corresponding capacities for the 5-chunk signs.

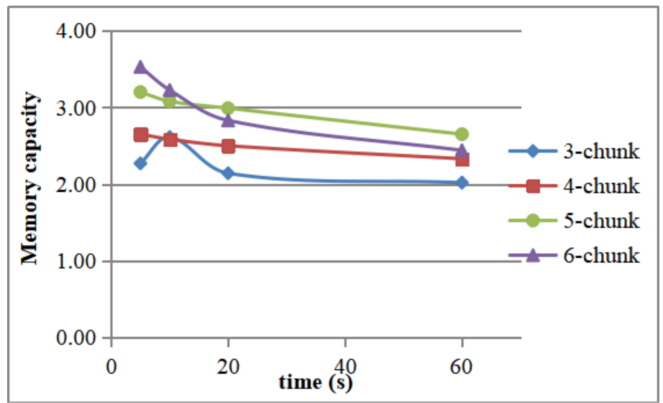

(a) entrance sign

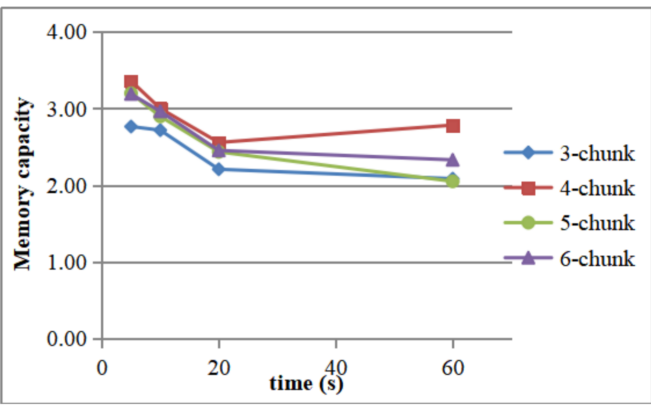

(b) exit sign

Figure 7. Short-term memory capacity.

When the type of information in the sign was more complex (exit sign), subjects' maximum STM capacity at $5 \mathrm{~s}, 10 \mathrm{~s}, 20 \mathrm{~s}$, and $60 \mathrm{~s}$ was 3.36, 3.00, 2.56, and 2.78, respectively. These maximum memory capacities appeared throughout all four different signs by the number of information chunks (i.e., 3-, 4-, 5-, and 6-chunk signs) and did not increase with the amount of information. Therefore, our results showed that signs can have their information filtered and combined based on the complexity of the information type.

When the sign information chunk type was relatively simple, the amount of information could be increased. According to the data, entrance signs showed the following general rule: the more information chunks, the greater the STM capacity. Nonetheless, subjects' memory capacities for the 5-chunk entrance signs at $20 \mathrm{~s}$ and $60 \mathrm{~s}$ were greater than for the 6-chunk entrance signs. Therefore, it is recommended to set the upper limit of token information type between five and six chunks. Notwithstanding this, when the sign contains a more complex type of information, it is more appropriate to set the amount of information to four chunks.

Furthermore, there are specific parts of our results that we would like to emphasize. In Figure 5a, people's accuracy to remember the 3-chunk signs at $10 \mathrm{~s}$ suddenly increased. This happened because the sign corresponding to this point did not contain the subway logo; instead, it had the line number, the exit number, and the subway station name. As the previous analysis showed, pedestrians tend to ignore logo information and have a high ability to remember number information. This indicates that the type of information affects the STM capacity of pedestrians.

Similarly, this sudden decrease occurred in the 4-chunk exit signs at $20 \mathrm{~s}$. This sign contained exit letters and the orientation information. As previously mentioned, people have a lower memory capacity for both types of information, which may explain the 4-chunk polyline of the exit sign showing to be concave at the $20 \mathrm{~s}$ position. This shows that the type of information chunk influenced people's STM capacity. Therefore, these are the suggestions we can gather from our results: in China, the orientation information can be avoided in subway signs; information should be numbered (letters should be avoided); and logo information is highly ignored by pedestrians, so it can be excluded from the sign when unnecessary. 


\section{Discussion}

Our results showed that pedestrians' STM ability to remember subway signs was related to the type, combination, and complexity of the information chunks. In terms of information type, pedestrians were shown to have the ability to remember numbers and arrow symbols but not orientation and letters. Yu et al.'s research on STM [21] showed similar results, and the author suggested that people tend to have better short-term memories related to information that is more familiar to them. Thus, numbers may be more familiar than letters to Chinese pedestrians in a subway station.

Regarding the combination of different information chunks, our results showed that pedestrians may have an improved STM capacity toward an entire sign if it contains information that is easier to remember, such as numbers and arrows. Conversely, pedestrians may have a reduced STM capacity toward an entire sign if it contains inconvenient information (e.g., orientation). These results may owe to pedestrians' lack of concentration; when there is information in the sign that is difficult to recall, the pedestrian may need to spend too much energy to remember it, subsequently hindering overall memory.

Finally, regarding information complexity, when we compared the content of the information that was present in both types of signs (entrance and exit) that had the same amount of information chunks (4-chunk), we found that the combination of the types of information was relatively simple in the entrance sign. Hence, for entrance signs, pedestrians' STM capacity was higher.

It is also important to note that the study of STM in subway signs has a lot in common with other studies on the topic. According to the attenuation characteristics of STM law $[10,11]$, the rate of forgetting increases with time. In our study, the results at the $5 \mathrm{~s}$ time period regarding STM capacity should demonstrate pedestrians' largest memory capacity. Our results showed that, for the entrance sign, the maximum value for STM capacity at $5 \mathrm{~s}$ was 3.53 ; for the exit sign, it was 3.36 . At $20 \mathrm{~s}$, for the entrance sign, it was 2.99; for the exit sign, it was 2.56. Moreover, these results were similar to those of Cowan and Kaplan [13-15], who suggested that people's STM can hold three to five information chunks at once. Nonetheless, the results of the cited study were slightly higher, and the difference between results may owe to the use of different research stimuli and information chunk types; in addition, the information combinations in their study were simpler.

Regarding different information types, Alvarez and Cavanagh found that information types had an impact on STM capacity [19]; after examining people's STM for different types of information, they found that people's memory capacity varied from 1.6 to 4.4. Nonetheless, the results of Chen may be most closely related to ours; he took four kinds of information as experimental stimuli: Chinese characters, figures, numbers, and position. One set of stimuli was shown at a time through a slide show, and the subjects then recalled and wrote down the sequence of items. Results showed that people's STM ability was the largest for numbers, followed by figures, Chinese characters, and position [23]. Although the objects and methods of Chen's study were different from those of ours, our data results were somewhat consistent.

Therefore, the following practical suggestions can be made for subway station sign designers:

1. Pedestrians' memory capacity regarding subway signs gradually decreases as time goes by. Considering that STM capacity changes with time, it seems reasonable to refresh pedestrians' memory every $20 \mathrm{~s}$. Through the literature analysis of pedestrian walking speed, we find that pedestrians of different ages have different walking speeds for different travel purposes, about 1.0-1.5 m/s [33-37], with older pedestrians usually having a lower speed. The setting of subway signs should give full consideration to the information needs of pedestrians with slow walking speed, so we take $1.0 \mathrm{~m} / \mathrm{s}$ as the lower limit of the walking speed and multiply it with the $20 \mathrm{~s}$ time interval of information retelling. Hence, we suggest that path-finding signs should be installed at every $20 \mathrm{~m}$ to improve pedestrians' passage efficiency within Chinese subway stations. 
2. Use numbers to code the lines and exits, as they were shown to be easier for Chinese pedestrians to remember. Avoid the use of letters, as they showed the opposite outcome.

3. Avoid using unnecessary logos and orientation information in order to decrease the total number of information chunks on the subway sign. Through the research of this paper, we found that when the information composition of signs was relatively complex, the total number of information chunks would affect the pedestrian's memory. An analysis of the existing signs shows that they often contain some miscellaneous information, such as logos with repeated text meanings and orientation information, which pedestrians pay less attention to. It is more convenient for pedestrians to obtain and remember the required information by appropriately reducing the number of information chunks. Less miscellaneous information makes it easier for pedestrians to get the information they need, rather than being disturbed by other unnecessary information.

In this research, we studied the influence of the hanging entrance signs and hanging exit signs on pedestrians' STM characteristics. In future, more subway sign types (e.g., ground type, attached type, standing type, and transfer signs) can be investigated. Meanwhile, the indoor test was adopted to eliminate the interference of the pedestrian flow and ensure the smooth progress of the test process. Through further research, the results can be put into practice using real subway signs for verification.

\section{Conclusions}

A large number of scholars have studied STM from various perspectives (e.g., physiology, object comparison between Chinese and English studies, etc.). However, few have analyzed people's STM for multiple types of information and in real environments. Similarly, there are few practical studies on this subject. In subway stations, signage is an important tool to ensure efficient pedestrian circulation and traffic. Thus, this paper analyzed the characteristics of pedestrians' STM and pedestrians' law of STM capacity in a subway environment; we did so by carrying out a cognitive test in which we utilized photographs of real subway signs as the stimuli. We believe our research results may provide theoretical support and technical guidance for the design and layout of subway station signs in China. The main findings were as follows:

1. Entrance and exit subway signs found throughout China had their information characteristics summarized.

2. Different types of information chunks contained in subway signs evoked different memory capacities among pedestrians. Generally speaking, the order, from high to low, for people's ability to accurately remember different types of information chunks was numerals $\approx$ arrow $>$ location $>$ letter $>\log 0 \approx$ orientation.

3. Pedestrians' STM ability was related to the number of information chunks, the combination of the information chunks, and the complexity of the information contained in the sign. When the information type was simple and familiar, the number of information chunks had little effect on pedestrians' STM capacity. When the information type was complex and/or unfamiliar, the number of information chunks had a greater effect, and pedestrians' STM capacity reached its peak when there were four information chunks.

In conclusion, we provided suggestions for the design and layout of subway signs in China, which we believe could improve the quality of the subway station services as well as promote sustainable development from the perspective of improving human travel quality and creating a better travel environment.

Author Contributions: Conceptualization, H.L. and J.X.; methodology, H.L.; software, H.L.; validation, H.L. and X.Z.; formal analysis, H.L.; investigation, H.L. and F.M.; resources, J.X.; data curation, H.L.; writing-original draft preparation, H.L.; writing-review and editing, J.X. and X.Z.; visualization, H.L. and F.M.; supervision, J.X.; project administration, J.X. All authors have read and agreed to the published version of the manuscript. 
Funding: This research was funded by the Scientific Research Project of the Transportation Department of Shaanxi Province (16-40 K).

Institutional Review Board Statement: The study was conducted according to the guidelines of the Declaration of Helsinki, and approved by the Institutional Review Board of School of Highway, Chang'an University. (date of approval: 2020.5.8).

Informed Consent Statement: Informed consent was obtained from all subjects involved in the study. Data Availability Statement: Not applicable.

Acknowledgments: The opportunity to research this topic is made possible by funding provided by the Scientific Research Project of the Transportation Department of Shaanxi Province. We thank the pedestrians for their cooperation during the test.

Conflicts of Interest: The authors declare no conflict of interest.

\section{References}

1. Atkinson, R.C.; Shiffrin, R.M. Human Memory: A Proposed System and its Control Processes. Psychol. Learn. Motiv. 1968, 2, 89-195.

2. Miller, C.E. The Effect of Inter-Stimulus Competition on Visual Short-Term Memory Capacity. Ph.D. Thesis, Bangor University, Wales, UK, 2016.

3. Luck, S.J.; Vogel, E.K. Visual Working Memory Capacity: From Psychophysics and Neurobiology to Individual Differences. Trends Cogn. Sci. 2013, 17, 391-400. [CrossRef] [PubMed]

4. Brady, T.F.; Konkle, T.; Alvarez, G.A. A Review of Visual Memory Capacity: Beyond Individual Items and toward Structured Representations. J. Vis. 2011, 11, 4. [CrossRef] [PubMed]

5. Bettencourt, K.C.; Xu, Y. Decoding the Content of Visual Short-term Memory under Distraction in Occipital and Parietal Areas. Nat. Neurosci. 2016, 19, 150-157. [CrossRef]

6. Stokes, M.G.; Kusunoki, M.; Sigala, N.; Nili, H.; Gaffan, D.; Duncan, J. Dynamic Coding for Cognitive Control in Prefrontal Cortex. Neuron 2013, 78, 364-375. [CrossRef]

7. Riley, M.R.; Constantinidis, C. Role of Prefrontal Persistent Activity in Working Memory. Front. Syst. Neurosci. 2015, 9, 181. [CrossRef]

8. Stokes, M.G. 'Activity-silent' Working Memory in Prefrontal Cortex: A Dynamic Coding Framework. Trends Cogn. Sci. 2015, 19, 394-405. [CrossRef]

9. Kane, M.J.; Engle, R.W. Working-memory Capacity and the Control of Attention. J. Exp. Psychol. Gen. 2003, 132, 47-70. [CrossRef]

10. Peterson, L.R.; Peterson, M.J. Short-term Memory Retention of Individual Verbal Items. J. Exp. Psychol. 1959, 193. [CrossRef]

11. Gerrig, R.J.; Zimbardo, P.; Svartdal, F.; Brennen, T.; Donaldson, R.; Archer, T. Psychology and Life, 16th ed.; Pearson: London, UK, 2012.

12. Miller, G.A. The Magical Number Seven Plus or Minus Two: Some Limits on Our Capacity for Processing Information. Psychol. Rev. 1994, 101, 343-352. [CrossRef]

13. Cowan, N. The Magical Number 4 in STM: A Reconsideration of Mental Storage Capacity. Behav. Brain Sci. 2001, 24, 87-114. [CrossRef]

14. Cowan, N. Working Memory Capacity. Curr. Dir. Psychol. Sci. 2005, 19, 51-57. [CrossRef]

15. Kaplan, S.; Kaplan, R. Arousal and Memory: A Comment. Psychon. Sci. 1968, 10, 291-292. [CrossRef]

16. Luck, S.J.; Vogel, E.K. The capacity of visual working memory for features and conjunctions. Nature 1997, 390, 279. [CrossRef]

17. Sligte, I.G.; Vandenbroucke, A.R.E.; Scholte, H.S.; Lamme, V.A.F. Detailed Sensory Memory, Sloppy Working Memory. Front. Psychol. 2010, 1, 175. [CrossRef]

18. Sligte, I.G.; Scholte, H.S.; Lamme, V. Are There Multiple Visual Short-term Memory Stores? PLoS ONE 2008, 3, e1699. [CrossRef]

19. Alvarez, G.A.; Cavanagh, P. The Capacity of Visual Short Term Memory is Set Both by Visual Information Load and by Number of Objects. Psychol. Sci. 2004, 15, 106-111. [CrossRef]

20. Simon, H.A. How Big Is a Chunk?: By Combining Data From Several Experiments, a Basic Human Memory Unit Can be Identified and Measured. Science 1974, 183, 482-488. [CrossRef]

21. Yu, B.L.; Jing, Q.C. Short-term Memory Span of Chinese Words. J. Psychol. 1985, 17, 361-368.

22. Yu, B.L.; Zhang, W.T.; Jing, Q.C.; Peng, R.X.; Simon, H.A. STM Capacity for Chinese and English Language Materials. Mem. Cogn. 1985, 13, 202-207. [CrossRef]

23. Chen, H. Age and Material Characteristics of Short-term Memory Capacity. J. Tianjin Norm. Univ. Soc. Sci. Ed. 1988, 4, 25-30.

24. Kevin, L. Reconsidering the Image of the City. In Cities of the Mind; Lloyd, R., Robert, M.H., Eds.; Spring: Naperville, IL, USA, 1984; pp. 151-161.

25. Arthur, P.; Passini, R. Wayfinding: People, Signs, and Architecture; McGraw-Hill Book Co.: New York, NY, USA, 1992.

26. Nassa, K. Sign Visibility for Pedestrians Assessed with Agent-Based Simulation. Transp. Res. Rec. J. Transp. Res. Board 2011, 2264, 18-26. [CrossRef] 
27. Vilar, E.; Rebelo, F.; Noriega, P.; Duarte, E.; Mayhorn, C.B. Effects of Competing Environmental Variables and Signage on Route-choices in Simulated Everyday and Emergency Wayfinding Situations. Ergonomics 2014, 57, 511. [CrossRef]

28. Wang, W.L.; Lo, S.M.; Liu, S.B.; Kuang, H. Microscopic Modeling of Pedestrian Movement Behavior: Interacting with Visual Attractors in the Environment. Transp. Res. Part C 2014, 44, 21-33. [CrossRef]

29. Beck, R. Designing for Passenger Information Needs in Subway Systems. In Transit Research Developments; Transportation Research Board: Washington, DC, USA, 1984; pp. 17-24.

30. Wei, L.Y.; Xu, J.L.; Jia, X.L.; Zhang, X.D.; Li, H.R. Effects of Safety Facilities on Driver Distance Perception in Expressway Tunnels. J. Adv. Transp. 2018. [CrossRef]

31. Rhodes, M.G.; Castel, A.D. Memory Predictions Are Influenced by Perceptual Information: Evidence for Metacognitive Illusions. J. Exp. Psychol.Gen. 2008, 137, 615-625. [CrossRef] [PubMed]

32. Brown, J. Some Tests of the Decay Theory of Immediate Memory. Q. J. Exp. Psychol. 1958, 58, 12-21. [CrossRef]

33. Franek, M.; Rezny, L. Analysis of Factors Affecting Variations in Walking Speed in Urban Environment with Natural Elements. Ceskoslov. Psychol. 2014, 58, 14-30.

34. Zhao, Y.; Wu, T.; Wei, Y. Effects of Starting Position, Distance and Ending Point in a Walking Speed Test among Older Adults. Geriatr. Gerontol. Int. 2020, 20, 680-684. [CrossRef]

35. Aghabayk, K.; Parishad, N.; Shiwakoti, N. Investigation on the Impact of Walkways Slope and Pedestrians Physical Characteristics on Pedestrians Normal Walking and Jogging Speeds. Saf. Sci. 2021, 133, 105012. [CrossRef]

36. Lam, W.; Cheung, C.Y. Pedestrian Speed/Flow Relationships for Walking Facilities in Hong Kong. J. Transp. Eng. 2000, 126, 343-349. [CrossRef]

37. Lei, B.; Xu, J.L.; Li, M.H.; Li, H.R.; Li, J.; Cao, Z.; Hao, Y.R.; Zhang, Y. Enhancing Role of Guiding Signs Setting in Metro Stations with Incorporation of Microscopic Behavior of Pedestrians. Sustainability 2019, 11, 6109. [CrossRef] 